\title{
On the Latent Roots of Compound Determinants and Brill's Determinants.
}

\author{
By Professor E. T. Whittaker.
}

(Read 10th November 1916. Received 21st November 1916.)

\section{§1. Introduction.}

The roots of the equation in $\lambda$

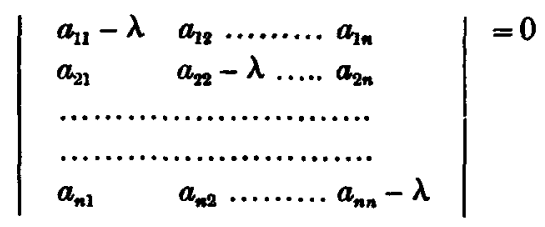

were named by Sylvester the latent roots of the determinant $\left|a_{n 1} a_{2 z} \ldots a_{n n}\right|$. So early as 1852, Sylvester showed* that if any determinant $D$ is given, we can at once write down a determinant whose latent roots are the squares of the latent roots of $D:$ this determinant is in fact the square of $D$, the process of squaring being performed by multiplying rows into columns : so that, e.g. if the latent roots of $\left|\begin{array}{ll}a & b \\ c & d\end{array}\right|$ are $\lambda_{1}$ and $\lambda_{2}$, then the roots of $\left|\begin{array}{ll}a^{2}+b c & a b+b d \\ a c+c d & b c+d^{2}\end{array}\right|$ are $\lambda_{1}{ }^{2}$ and $\lambda_{2}{ }^{2}$. Spottiswoode had also shown $\dagger$ in 1851 that the latent roots of the reciprocal of a determinant are the reciprocals of the latent roots of the determinant itself. Both these theorems were soon found to be particular cases of a general theorem which was enunciated by Sylvester thus: The latent roots of any function of a matrix are respectively the same functions of the latent roots of the matrix itself.

* First in connexion with axisymmetric determinants, in Nouv. Ann. 1852 : Coll. Papers 1, p. 364.

$\dagger$ Elementary theorems relating to determinants, London, 1851. 
In order to complete the definition of the terms used in the title of this paper, we must now explain what compound determinants and Brill's determinants are.

First, as regards compound determinants. Let $D$ be any determinant of order $n$. Consider all those minors of $D$ which are of order $m$ : they are in number $\left(\begin{array}{l}n \\ m\end{array}\right)^{2 *}$ : and arrange them so that in any one row (or column) those minors stand which are contained in the same $m$ rows (or columns) of $D$. The determinant thus formed, which was first considered by Cauchy, $\dagger$ is called the $m^{\text {th }}$ compound of $D$. The principal known theorem regarding compound determinants is due to Sylvester, $\ddagger$ and asserts that the $m^{\text {th }}$ compound of any determinant $D$ is equal to

$$
D^{(m-1)}
$$

Next, as regards Brill's determinants. In 1870 , Brill, $\S$ in the course of some investigations regarding those members of a pencil of curves, which touch a given curve, came upon the determinant

$$
\left|\begin{array}{llllll}
x_{1}^{2} & x_{2}{ }^{2} & x_{3}{ }^{2} & 2 x_{2} x_{3} & 2 x_{3} x_{1} & 2 x_{1} x_{2} \\
y_{1}{ }^{2} & y_{2}{ }^{2} & y_{3}{ }^{2} & 2 y_{2} y_{3} & 2 y_{3} y_{1} & 2 y_{1} y_{2} \\
z_{1}{ }^{2} & z_{2}{ }^{2} & z_{3}{ }^{2} & 2 z_{2} z_{3} & 2 z_{3} z_{1} & 2 z_{3} z_{2} \\
y_{1} z_{1} & y_{2} z_{2} & y_{3} z_{3} & y_{3} z_{3}+z_{2} y_{3} & y_{3} z_{1}+z_{3} y_{1} & y_{1} z_{2}+z_{1} y_{2} \\
z_{1} x_{1} & z_{2} x_{2} & z_{3} x_{3} & z_{2} x_{3}+x_{2} z_{3} & z_{3} x_{1}+x_{3} z_{1} & z_{1} x_{2}+x_{1} z_{2} \\
x_{1} y_{1} & x_{2} y_{2} & x_{3} y_{3} & x_{2} y_{3}+y_{2} x_{3} & x_{3} y_{1}+y_{3} x_{1} & x_{1} y_{2}+y_{1} x_{2}
\end{array}\right|
$$

which he proved equal to

$$
\left|\begin{array}{lll}
x_{1} & x_{2} & x_{3} \\
y_{1} & y_{2} & y_{3} \\
z_{1} & z_{2} & z_{3}
\end{array}\right|
$$

* $\left(\begin{array}{l}n \\ m\end{array}\right)$ means the number of combinations of $n$ things $m$ at a time, i.e. $\frac{n !}{m !(n-m) !}$.

† Journal de l'Ec. Pol., cah. 17, p. 93.

$\ddagger$ Phil. Mag. (4) 1 (1851), pp. 295, 415.

8 Math. Ann. 3, p. 458. 
It is evident that this determinant of Brill's is the eliminant of the $\theta$ 's between the equations

$$
\left\{\begin{array}{r}
\left(x_{1} \theta_{1}+x_{2} \theta_{2}+x_{3} \theta_{3}\right)^{2}=0 \\
\left(y_{1} \theta_{1}+y_{2} \theta_{2}+y_{3} \theta_{3}\right)^{2}=0 \\
\left(z_{1} \theta_{1}+z_{2} \theta_{2}+z_{3} \theta_{3}\right)^{2}=0 \\
\left(y_{1} \theta_{1}+y_{2} \theta_{2}+y_{3} \theta_{3}\right)\left(z_{1} \theta_{1}+z_{2} \theta_{2}+z_{3} \theta_{3}\right)=0 \\
\left(z_{1} \theta_{1}+z_{2} \theta_{2}+z_{3} \theta_{3}\right)\left(x_{1} \theta_{2}+x_{2} \theta_{2}+x_{3} \theta_{3}\right)=0 \\
\left(x_{1} \theta_{1}+x_{2} \theta_{2}+x_{3} \theta_{3}\right)\left(y_{1} \theta_{1}+y_{2} \theta_{2}+y_{3} \theta_{3}\right)=0
\end{array}\right.
$$

and that it is therefore only one of an infinite set of determinants which may be found by taking a set of linear equations

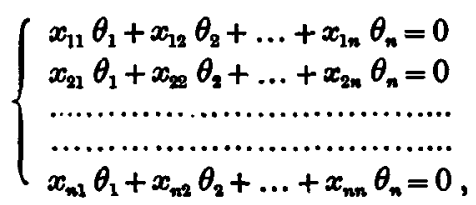

forming all combinations of these equations which are homogeneous of degree $m$ in the $\theta$ 's, and then eliminating the $\theta$ 's determinantally. Brill's original determinant given above represents the case $n=3, m=2$. When there are $n$ variables $\theta_{1}, \theta_{2}, \ldots \theta_{n}$, and the linear equations are raised to the power $m$, we shall call the deter-

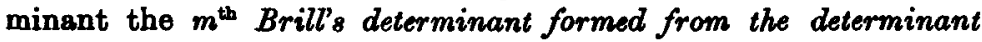
$\left|x_{11} x_{22} x_{23} \ldots x_{n=1}\right|$.

The object of the present paper is to find the latent roots of all compound determinants and Brill's determinants, in terms of the latent roots of the determinant from which they are formed.

\section{\$2. The latent roots of compound determinants.}

We propose to show first that the latent roots of the $m^{\text {th }}$ compound of a determinant $D$ are the products, $m$ at a time, of the latent roots of $D$. To prove this, suppose that $\lambda_{1}, \lambda_{2}, \ldots \lambda_{m}$ are $m$ distinct latent roots of a determinant $D=\left|a_{11} a_{22} a_{33}, \ldots a_{n n}\right|:$ so that the values of $\left(x_{11}, x_{12}, \ldots x_{1 n}\right)$, which are not all zero, can be found to satisfy the $n$ equations

$$
a_{p 1} x_{11}+a_{p 2} x_{12}+\ldots+a_{p n} x_{1 n}=\lambda_{1} x_{1 p} \quad(p=1,2, \ldots n)
$$

and values of $\left(x_{21}, x_{22}, \ldots, x_{2 n}\right)$, which are not all zero and are 
not all identical respectively with $\left(x_{11}, x_{12}, \ldots x_{1 n}\right)$, can be found to satisfy the $n$ equations

$$
a_{p 1} x_{21}+a_{p 2} x_{22}+\ldots+a_{p n} x_{2 n}=\lambda_{2} x_{2 p} \quad(p=1,2, \ldots n) \ldots \ldots
$$

and so on. These equations (1), (2), etc., show that the determinant

$$
\lambda_{1} \lambda_{2} \ldots \lambda_{m}\left|x_{11} x_{22} \ldots x_{m m}\right|
$$

is the product of the two arrays

and

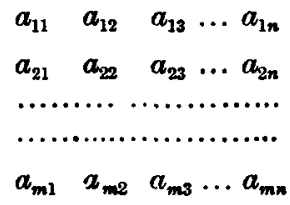

$$
\begin{aligned}
& x_{11} \quad x_{12} \quad x_{13} \ldots x_{1 n} \\
& x_{21} \quad x_{22} \quad x_{23} \ldots x_{2 n} \\
& \text {....................... } \\
& \begin{array}{lllll}
x_{m 1} & x_{m 2} & x_{m 3} \ldots x_{m n}
\end{array}
\end{aligned}
$$

and is therefore equal to

a sum of products of determinants formed from

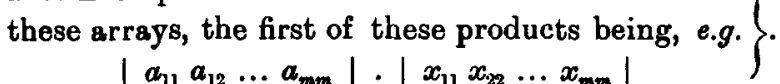

Equating (3) to (4), we have a linear equation

$$
\lambda_{1} \lambda_{2} \ldots \lambda_{m} X_{1}=A_{1} X_{1}+A_{2} X_{2}+A_{3} X_{3}+\ldots
$$

in which the variables $X_{1}, X_{2}, X_{3}, \ldots$ are all the $\left(\begin{array}{l}n \\ m\end{array}\right)$ determinants that can be formed by taking $m$ columns of the array

$$
\begin{aligned}
& x_{11} x_{12} \ldots x_{1 n} \\
& x_{21} x_{22} \ldots x_{2 n} \\
& \ldots \ldots \ldots \ldots \ldots \ldots \\
& \ldots \ldots \ldots \ldots \ldots . . . \\
& x_{m 1} x_{m 2} \ldots x_{m n},
\end{aligned}
$$

while the coefficients $A_{1}, A_{2}, A_{3}, \ldots$ are all the $\left(\begin{array}{l}n \\ m\end{array}\right)$ minors that can be formed from the first $m$ lines of the determinant $\left|a_{11} a_{22} a_{23} \ldots a_{n n}\right|$. 
In the same way we may obtain other linear equations in which the variables are the same quantities $X_{1}, X_{2}, X_{3}, \ldots$, and the coefficients are (on the left-hand side) $\lambda_{1} \lambda_{2} \ldots \lambda_{m}$ and (on the right-hand side) all the $\left(\begin{array}{l}n \\ m\end{array}\right)$ determinants that can be formed from some other $m$ lines of the determinant $\left|a_{11} a_{22} \ldots a_{m n}\right|$. We obtain altogether $\left(\begin{array}{l}n \\ m\end{array}\right)$ of these linear equations. Since the variables $X_{1}, X_{2}, X_{3}, \ldots$ in these equations are not all zero, we may eliminate them in the usual way, by equating to zero the determinant of the coefficients. But it is obvious that this determinant is simply the $m^{\text {th }}$ compound of $D$, with the quantity $\lambda_{1} \lambda_{2} \ldots \lambda_{m}$ subtracted from each term in the principal diagonal. Therefore the quantity $\lambda_{1} \lambda_{2} \ldots \lambda_{m}$ is one of the latent roots of the $m^{\text {th }}$ compound of $D$. Similarly, any other product of $m$ distinct latent roots of $D$ is one of the latent roots of the $m^{\text {th }}$ compound of $D$. But when the latent roots of $D$ are all distinct, the number of distinct products of this kind is $\left(\begin{array}{l}n \\ m\end{array}\right)$ : and the number of latent roots of the $m^{\text {th }}$ compound is equal to its order, which is also $\left(\begin{array}{l}n \\ m\end{array}\right)$. Therefore the latent roots of the $m^{\text {th }}$ compound of $D$ are precisely the products, $m$ at a time, of the latent roots of $D . *$ The principle of continuity shows that this statement is still true even if the latent roots of $D$ are not all distinct.

It may be remarked that this result furnishes a simple proof of Sylvester's theorem on compound determinants. For any determinant is evidently equal to the product of its own latent roots : so if $\Delta$ denotes the $m^{\text {th }}$ compound of $D$, we have $D$ equal to the product of its own latent roots, and $\Delta$ equal to the product of the $\left(\begin{array}{l}n \\ m\end{array}\right)$ products of these roots taken $m$ at a time. Now, of the products of the latent roots taken $m$ at a time, the number which

* Professor Metzler, in Amer. Jour. of Math. 16 (1894), p. 131, in the course of resesrohes on the minors of compound determinants, placed on record a saspicion of this theorem, but epparently did not pureue the matter further. 
contain any particular latent root is $\left(\begin{array}{c}n-1 \\ m-1\end{array}\right):$ and therefore we have

$$
\Delta=D^{\left(\begin{array}{c}
n-1 \\
m-1
\end{array}\right)},
$$

which is precisely Sylvester's formula.

We may note also that the theorem determines at once the number of latent roots of the $m^{\text {th }}$ compound which become equal to each other when any number of latent roots of the original determinant are equal.

\section{\$. The latent roots of Brill's determinants.}

We shall next show that the latent roots of the $m^{\text {th }}$ Brill's determinant formed from a determinant $D$ are the products $m$ at a time of the latent roots of $D$, when repetitions are allowed. The meaning of the phrase "when repetitions are allowed" may be illustrated by taking the case $n=3$, in which case the latent roots of $D$ may be denoted by $\lambda_{1}, \lambda_{2}, \lambda_{3}$; the products two at a time when repetitions are not allowed are $\lambda_{2} \lambda_{3}, \lambda_{3} \lambda_{1}, \lambda_{1} \lambda_{2}$; while the products two at a time when repetitions are allowed are $\lambda_{1}^{2}, \lambda_{2}^{2}, \lambda_{3}^{2}, \lambda_{2} \lambda_{3}, \lambda_{3} \lambda_{1}, \lambda_{1} \lambda_{2}$.

To prove the theorem, suppose that $\lambda_{1}, \lambda_{2}, \ldots, \lambda_{m}$ are any $m$ (the same or different) latent roots of a determinant $D=\left|a_{11} a_{22} \ldots a_{n n}\right|:$ so that values of $x_{11}, x_{12}, \ldots x_{1 n}$ which are not all zero can be found to satisfy the $n$ equations

$$
a_{p 1} x_{11}+a_{p 2} x_{18}+\ldots+a_{p n} x_{1 n}=\lambda_{1} x_{1 p} \quad(p=1,2, \ldots, n),
$$

and values of $x_{21}, x_{22}, \ldots, x_{2 n}$ which are not all zero (but which may be identical with $x_{11}, x_{12}, \ldots, x_{n 1}$ ) can be found which satisfy the $n$ equations

$$
a_{p 1} x_{n 1}+a_{p 2} x_{22}+\ldots+a_{p n} x_{2 n}=\lambda_{2} x_{2 p} \quad(p=1,2, \ldots, n),
$$

and so on.

Now form the continued product of any one of the equations (1), any one of the equations (2), and so on. We thus obtain an equation which is homogeneous of degree $m$ in the $x$ 's, and has on 
its left hand side (say)

$$
\lambda_{1} \lambda_{2} \lambda_{3} \ldots \lambda_{m} x_{1 p} x_{2 p} x_{3 r} \ldots
$$

Add to this the similar equations in which the second suffixes $p, q, r, \ldots$ are permuted in every possible way. We thus obtain an equation which we may write

$$
\lambda_{1} \lambda_{2} \lambda_{3} \ldots \lambda_{m} X_{k}=A_{1} X_{1}+A_{2} X_{2}+A_{3} X_{3}+\ldots,
$$

where $X_{1}, X_{2}, X_{3}, \ldots$ are functions homogeneous of degree $m$ in the $x$ 's, which are symmetric as between the different sets of $x^{\prime}$ 's; e.g. in the case $n=3, m=2$, they would be the 6 quantities

$x_{11} x_{21}, x_{12} x_{22}, x_{13} x_{23},\left(x_{12} x_{23}+x_{13} x_{22}\right),\left(x_{11} x_{23}+x_{13} x_{21}\right),\left(x_{11} x_{22}+x_{12} x_{21}\right)$, and the coefficients $A_{1}, A_{2}, A_{3}, \ldots$ are the quantities which occur in some one row of the $m^{\text {th }}$ Brill's determinant formed from $D$.

If now we eliminate the $X$ 's determinantally between (3) and all other equations of the same type, we have a certain determinant equal to zero: and this determinant is simply the $m^{\text {th }}$ Brill's determinant of $D$ with $\lambda_{1} \lambda_{2} \ldots \lambda_{m}$ subtracted from each term in the principal diagonal. This shows that $\lambda_{1} \lambda_{2} \ldots \lambda_{m}$ is one of the latent roots of the $\boldsymbol{m}^{\text {th }}$ Brill's determinant of $D$, where $\lambda_{1}, \lambda_{2}, \ldots \lambda_{m}$ are any $m$ (the same or different) latent roots of $D$; whence the theorem enunciated above follows readily. of $D$.

\$4. A further determinant whose latent roots depend on those

If, instead of forming equation (3) of $\S 3$ by adding similar equations in which the second suffixes are permuted, we had left these equations in their original form, they would have constituted a set of equations linear in variables $Y_{1}, Y_{3}, Y_{3}, \ldots$ where $Y_{1}, Y_{2}, Y_{3}, \ldots$ are all the $n^{m}$ products which can be formed by taking one factor from the set $\left(x_{11}, x_{12}, \ldots, x_{1 n}\right)$, one factor from the set $\left(x_{21}, x_{22}, \ldots, x_{2 n}\right)$, and so on : e.g. in the case $n=3, m=2$, $Y_{1}, Y_{2}, Y_{3} \ldots$ are the 9 quantities

$x_{11} x_{21}, x_{11} x_{22}, x_{11} x_{23}, x_{12} x_{21}, x_{12} x_{22}, x_{12} x_{23}, x_{13} x_{21}, x_{13} x_{22}, x_{13} x_{23}$.

If we eliminate $Y_{1}, Y_{2}, Y_{3}, \ldots$ from the equations determinantally, we have zero equal to a determinant of order $n^{m}$, which has 
$\lambda_{1} \lambda_{2} \ldots \lambda_{m}$ subtracted from every element in its principal diagonal : so that $\lambda_{1} \lambda_{2} \ldots \lambda_{m}$ is one of the latent roots of this determinant, which we call $\Delta$. It may readily be shown that the latent roots of $\Delta$ are the same as those of the $m^{\text {th }}$ Brill's determinant of $D$, namely, all the products $m$ at a time of the latent roots of $D$, when repetitions are allowed: but that some of these latent roots of the Brill's determinant are. repeated roots of $\Delta$. Thus in the case $n=3, m=2$, the latent roots of $\Delta$, which is in this case of the 9 th order, are

$\lambda_{1}^{2}, \lambda_{2}^{2}, \lambda_{3}^{2}, \lambda_{2} \lambda_{3}$ repeated, $\lambda_{3} \lambda_{1}$ repeated, and $\lambda_{1} \lambda_{2}$ repeated, where $\lambda_{1}, \lambda_{2}, \lambda_{3}$, are the latent roots of the original determinant $D$. 